\title{
Epidemiology of vampire bat-transmitted rabies virus in Goiás, central Brazil: re-evaluation based on G-L intergenic region
}

\author{
Shinji Hirano', Takuya Itou ${ }^{1 *}$, Adolorata AB Carvalho², Fumio H Ito ${ }^{3}$, Takeo Sakai ${ }^{1}$
}

\begin{abstract}
Background: Vampire bat related rabies harms both livestock industry and public health sector in central Brazil. The geographical distributions of vampire bat-transmitted rabies virus variants are delimited by mountain chains. These findings were elucidated by analyzing a high conserved nucleoprotein gene. This study aims to elucidate the detailed epidemiological characters of vampire bat-transmitted rabies virus by phylogenetic methods based on 619-nt sequence including unconserved G-L intergenic region.

Findings: The vampire bat-transmitted rabies virus isolates divided into 8 phylogenetic lineages in the previous nucleoprotein gene analysis were divided into 10 phylogenetic lineages with significant bootstrap values. The distributions of most variants were reconfirmed to be delimited by mountain chains. Furthermore, variants in undulating areas have narrow distributions and are apparently separated by mountain ridges.

Conclusions: This study demonstrates that the 619-nt sequence including G-L intergenic region is more useful for a state-level phylogenetic analysis of rabies virus than the partial nucleoprotein gene, and simultaneously that the distribution of vampire bat-transmitted RABV variants tends to be separated not only by mountain chains but also by mountain ridges, thus suggesting that the diversity of vampire bat-transmitted RABV variants was delimited by geographical undulations.
\end{abstract}

\section{Background}

Rabies is a zoonosis that kills infected mammals, including humans, and is mainly transmitted by carnivores. In the Americas, chiropterans (insectivorous, frugivorous and hematophagous bat) are another reservoir of this disease. Although dog-transmitted rabies in central Brazil has been reduced by aggressive vaccination programs [1], chiroptera (particularly the common vampire bat, Desmodus rotundas)-transmitted rabies remains endemic in this region, and harms both the livestock industry and the public health sector $[2,3]$.

To date, vampire bat-transmitted rabies in livestock has been controlled by reducing the population of vampire bats and by vaccinating livestock $[3,4]$. However, the depopulation of vampire bats has limitations and the effects are temporary, while vaccination of livestock is

\footnotetext{
* Correspondence: itou.takuya@nihon-u.ac.jp

'Nihon University Veterinary Research Center, 1866 Kameino, Fujisawa 2520880, Kanagawa, Japan

Full list of author information is available at the end of the article
}

only carried out for some animals and is ineffective in decreasing rabies levels in vampire bats.

For the sustainable and effective control of vampire bat rabies, further knowledge of epidemiological features, such as vampire bat ecology and the dynamics of vampire bat-transmitted rabies, is necessary. Molecular epidemiological analysis of vampire bat-transmitted cattle rabies cases using the partial nucleoprotein gene, which is the most conserved gene in the rabies virus (RABV) genome, has suggested that the distribution of variants in Brazil is delimited by mountain chains and clustered in tens of thousands of square kilometers [5]. However, the vampire bats migrate several kilometers from their nests [6]. To elucidate a more detailed genetic clustering and geo-distribution of genetic clades of vampire bat-transmitted RABV, 204 isolates from Goiás, which includes the 185 isolates analyzed previously, were employed and analyzed by a phylogenetic method based on a nucleotide sequence encompassing the G-L intergenic region locating between glycoprotein 
(G) and polymerase (L) gene loci, which is the most divergent region in the RABV genome and is used for monitoring epidemiological changes in the evolution of RABV $[7,8]$.

\section{Results}

\section{Phylogenetic analysis}

The 204 RABV isolates divided into 8 phylogenetic lineages in the previous nucleoprotein gene analysis were divided into 10 phylogenetic lineages with significant bootstrap values (Figure 1; details shown in Table 1). Isolates of the C-5 and C-6 lineages designated by Kobayashi et al. belonged to the A-lineage, while the B-lineage consisted of some isolates of C-5. The C-, D-, $\mathrm{F}$ - and J-lineages included isolates belonging to $\mathrm{C}-12$, $\mathrm{C}-1, \mathrm{C}-22$ and $\mathrm{C}-3$, respectively. Isolates of $\mathrm{C}-20$ belonged to the I-lineage, and $\mathrm{C}-21$ was divided into three lineages; G-, H- and I-lineages. Two isolates not belonging to any lineages in previous studies were assigned to the E-lineage.

\section{Geographical plotting}

In this study, geographical areas in Goiás were divided by mountain chains into the Northwest, North central, Northeast and South regions (Figure 2). Most isolates of the A-lineage were distributed in the South region. The B-lineage was likely to exist in the Northeast and North central regions. The isolates belonging to the C-, E-, F-, G-, $\mathrm{H}$ - and I-lineages were distributed in the Northwest region. The G-, H- and I-lineages have narrow distributions and are apparently separated by mountain ridges (Figure 2; Area I), while the A-lineage was distributed in a wide range throughout a Southeastern basin (Figure 2; Area II). The isolates of the D-lineage were plotted on an eastern edge of the North central region. The J-lineage had a wide geographical distribution in Goiás.

\section{Discussion}

Previous studies had elucidated that the distributions of vampire bat and transmitted RABV are delimited by mountain chains [5]. In the present study, it was reconfirmed that mountain chains divide the distribution patterns of each viral lineage. Furthermore, the isolates belonging to $\mathrm{C}-5$ having a wide range in Goiás were divided into the A- and B-lineages, and were found to be distributed in the South and North regions on either side of a mountain chain. This finding supports Kobayashi's hypothesis that distribution of vampire bat-transmitted RABV is affected by mountain chains.

The same variants of vampire bat-transmitted RABV were spread widely in flat low lands $(<800 \mathrm{~m})$, but at higher elevations (800-1600 m), they had a narrower distribution [9]. However, the G-, H- and I-lineages were found to be separated by mountain ridges in low

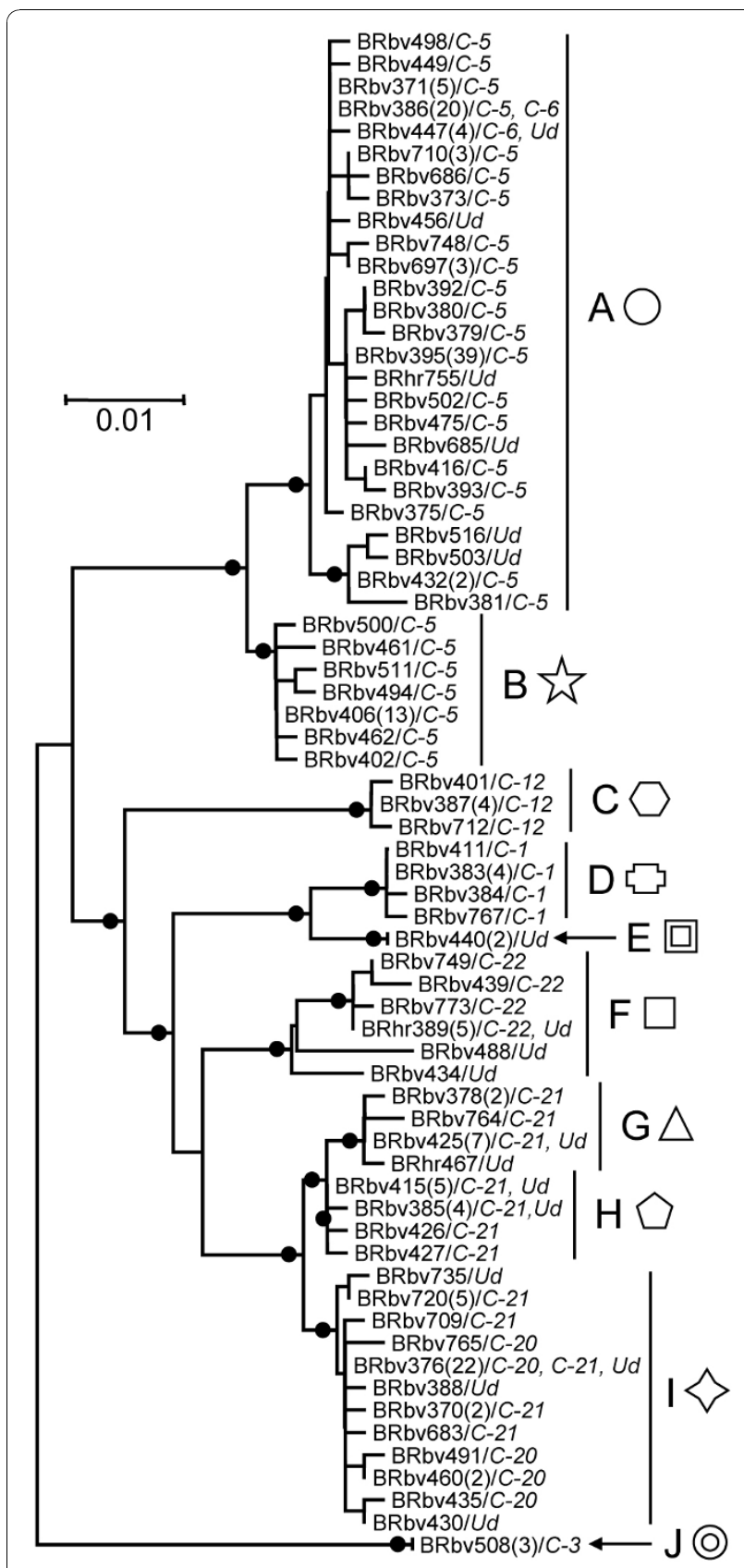

Figure $1 \mathrm{NJ}$ tree based on 619-nt sequence of the partial G and the G-L intergenic region. Full circles on internal branches indicate significant bootstrap values (>70\%). The number of isolates retaining $100 \%$ nucleotide similarity with the representative isolate is shown in parentheses. Lineages of the isolates defined in a previous study [5] are shown in italics after a diagonal (Ud: undefined).

areas $(400-800 \mathrm{~m})$ located in a southern undulating area of the Northwestern region (Figure 2; Area I). Furthermore, the A-lineage was located in an eastern basin of the South region (> $800 \mathrm{~m}$; Figure 2; Area II). Considering that the higher lands showed an undulating 
Table 1 Isolates from Goiás

\begin{tabular}{|c|c|c|c|c|c|c|c|}
\hline \multirow[b]{2}{*}{ Sample } & \multirow[b]{2}{*}{ Species } & \multirow[b]{2}{*}{ Location } & \multirow[b]{2}{*}{ Year } & \multicolumn{2}{|c|}{ Grouping } & \multicolumn{2}{|c|}{ Accession No. } \\
\hline & & & & This study & Previous studyc & N203-nt & Partial G \& GL \\
\hline BRbv371 & Cattle & Caldas Novas & 2002 & $A$ & $C-5$ & AB307182 & AB544082 \\
\hline BRbv372 & Cattle & Água Limpa & 2002 & A & $C-5$ & AB307183 & AB544083 \\
\hline BRbv373 & Cattle & Caldas Novas & 2002 & A & $C-5$ & AB307184 & AB544084 \\
\hline BRbv375 & Cattle & Santa Cruz de Goias & 2002 & A & $C-5$ & AB307186 & AB544085 \\
\hline BRbv379 & Cattle & Corumbaíba & 2002 & A & $C-5$ & AB307190 & AB544089 \\
\hline BRbv380 & Cattle & Corumbaíba & 2002 & A & $C-5$ & AB307191 & AB544090 \\
\hline BRbv381 & Cattle & Cristalina & 2002 & A & $C-5$ & AB307192 & AB544091 \\
\hline BRbv386 & Cattle & Ipameri & 2002 & $A$ & $C-5$ & AB307197 & AB544095 \\
\hline BRbv391 & Cattle & Caldas Novas & 2002 & A & $C-5$ & AB307201 & AB544099 \\
\hline BRbv392 & Cattle & Água Limpa & 2002 & $A$ & $C-5$ & AB307202 & AB544100 \\
\hline BRbv393 & Cattle & Itumbiara & 2002 & A & $C-5$ & AB307203 & AB544101 \\
\hline BRbv395 & Cattle & Itumbiara & 2002 & $A$ & $C-5$ & AB307205 & AB544102 \\
\hline BRbv396 & Cattle & Itumbiara & 2002 & A & $C-5$ & AB307206 & AB544103 \\
\hline BRbv397 & Cattle & Buriti Alegre & 2002 & A & $C-5$ & AB307207 & AB544104 \\
\hline BRbv398 & Cattle & Itumbiara & 2002 & A & $C-5$ & AB307208 & AB544105 \\
\hline BRbv400 & Cattle & Buriti Alegre & 2002 & $A$ & $C-5$ & AB307210 & AB544107 \\
\hline BRbv404 & Cattle & Buriti Alegre & 2002 & A & $C-5$ & AB307214 & AB544111 \\
\hline BRbv405 & Cattle & Buriti Alegre & 2002 & A & $C-5$ & AB307215 & AB544112 \\
\hline BRbv407 & Cattle & Goiandira & 2002 & A & $C-5$ & AB307217 & AB544114 \\
\hline BRbv412 & Cattle & Itapaci & 2002 & $A$ & $C-5$ & AB307222 & AB544118 \\
\hline BRbv413 & Cattle & Caldas Novas & 2002 & A & $C-5$ & AB307223 & AB544119 \\
\hline BRbv416 & Cattle & Morrinhos & 2002 & A & $C-5$ & AB307226 & AB544122 \\
\hline BRbv421 & Cattle & Nova Aurora & 2002 & A & $C-5$ & AB307231 & AB544124 \\
\hline BRbv429 & Cattle & Corumbaíba & 2002 & A & $U d^{a}$ & AB307238 & AB544129 \\
\hline BRbv432 & Cattle & Ipameri & 2002 & A & $C-5$ & AB307241 & AB544132 \\
\hline BRbv438 & Cattle & Buriti Alegre & 2002 & $A$ & $C-5$ & AB307247 & AB544137 \\
\hline BRhr441 & Horse & Buriti Alegre & 2002 & A & Ud & AB307251 & AB544140 \\
\hline BRbv442 & Cattle & Buriti Alegre & 2002 & $A$ & $C-5$ & AB307252 & AB544141 \\
\hline BRbv447 & Cattle & Urutaí & 2002 & A & $C-6$ & AB307255 & AB544143 \\
\hline BRbv449 & Cattle & Caldas Novas & 2002 & A & $C-5$ & AB307256 & AB544145 \\
\hline BRbv451 & Cattle & Ipameri & 2002 & $A$ & $C-5$ & AB307258 & AB544147 \\
\hline BRbv453 & Cattle & São Luis de Montes Belos & 2002 & A & $C-5$ & AB307260 & AB544149 \\
\hline BRbv456 & Cattle & Orizona & 2002 & $A$ & Ud & AB307263 & AB544150 \\
\hline BRbv457 & Cattle & Água Limpa & 2002 & A & $C-5$ & AB307264 & AB544151 \\
\hline BRbv458 & Cattle & Buriti Alegre & 2002 & $A$ & $C-5$ & AB307265 & AB544152 \\
\hline BRbv466 & Cattle & Itumbiara & 2002 & A & $C-5$ & AB307273 & AB544157 \\
\hline BRbv469 & Cattle & Ipameri & 2002 & A & $C-6$ & AB307275 & AB544159 \\
\hline BRbv471 & Cattle & Santa Cruz de Goias & 2002 & A & $C-6$ & AB307277 & AB544161 \\
\hline BRbv472 & Cattle & Ipameri & 2002 & A & $C-6$ & AB307278 & AB544162 \\
\hline BRbv473 & Cattle & Itumbiara & 2002 & A & $C-5$ & AB307279 & AB544163 \\
\hline BRbv475 & Cattle & Caldas Novas & 2002 & A & $C-5$ & AB307281 & AB544165 \\
\hline BRbv477 & Cattle & Itumbiara & 2002 & $A$ & $C-5$ & AB307283 & AB544166 \\
\hline BRbv478 & Cattle & Itumbiara & 2002 & A & $C-5$ & AB307284 & AB544167 \\
\hline BRbv482 & Cattle & Panamá & 2002 & A & $C-5$ & AB307288 & AB544170 \\
\hline BRhr483 & Horse & Panamá & 2002 & A & Ud & AB307291 & AB544171 \\
\hline BRbv486 & Cattle & Itumbiara & 2002 & $A$ & $C-5$ & AB307293 & AB544172 \\
\hline BRbv489 & Cattle & Itumbiara & 2002 & A & Ud & AB307295 & AB544174 \\
\hline BRbv493 & Cattle & Ipameri & 2002 & A & $C-6$ & AB307298 & AB544177 \\
\hline BRbv495 & Cattle & Panamá & 2002 & A & $C-5$ & AB307300 & AB544179 \\
\hline BRbv496 & Cattle & Panamá & 2002 & A & $C-5$ & AB307301 & AB544180 \\
\hline
\end{tabular}


Table 1 Isolates from Goiás (Continued)

\begin{tabular}{|c|c|c|c|c|c|c|c|}
\hline BRbv497 & Cattle & Itumbiara & 2002 & A & $C-5$ & AB307302 & AB544181 \\
\hline BRbv498 & Cattle & Bela Vista de Goiás & 2002 & A & $C-5$ & AB307303 & AB544182 \\
\hline BRbv502 & Cattle & Itumbiara & 2002 & A & $C-5$ & AB307306 & AB544186 \\
\hline BRbv503 & Cattle & Orizona & 2002 & A & Ud & AB307307 & AB544187 \\
\hline BRbv509 & Cattle & Buriti Alegre & 2002 & A & $C-5$ & AB307311 & AB544192 \\
\hline BRbv514 & Cattle & Panamá & 2002 & A & $C-5$ & AB307316 & AB544196 \\
\hline BRbv516 & Cattle & Orizona & 2002 & A & Ud & AB307318 & AB544198 \\
\hline BRbv521 & Cattle & Urutaí & 2002 & A & $N d^{b}$ & $\mathrm{Nd}$ & AB544201 \\
\hline BRbv524 & Cattle & Urutaí & 2002 & A & $C-6$ & AB307323 & AB544204 \\
\hline BRbv526 & Cattle & Panamá & 2002 & A & $C-5$ & AB307325 & AB544206 \\
\hline BRbv527 & Cattle & Campos Belos & 2002 & A & $C-5$ & AB307326 & AB544207 \\
\hline BRbv684 & Cattle & Ipameri & 2001 & A & $C-5$ & AB307412 & AB544211 \\
\hline BRbv685 & Cattle & Corumbaíba & 2001 & A & $\mathrm{Nd}$ & $\mathrm{Nd}$ & AB544212 \\
\hline BRbv686 & Cattle & Ipameri & 2001 & A & $C-5$ & AB307413 & AB544213 \\
\hline BRbv687 & Cattle & Corumbaíba & 2001 & A & $C-5$ & AB307414 & AB544214 \\
\hline BRbv690 & Cattle & Ipameri & 2001 & A & $C-5$ & AB307417 & AB544215 \\
\hline BRbv691 & Cattle & Ipameri & 2001 & A & $C-5$ & AB307418 & AB544216 \\
\hline BRbv694 & Cattle & Iporá & 2001 & A & $\mathrm{Nd}$ & $\mathrm{Nd}$ & AB544219 \\
\hline BRbv697 & Cattle & Campo Alegre de Goiás & 2001 & A & $C-5$ & AB307423 & AB544221 \\
\hline BRbv698 & Cattle & Campo Alegre de Goiás & 2001 & A & $C-5$ & AB307424 & AB544222 \\
\hline BRbv699 & Cattle & Campo Alegre de Goiás & 2001 & A & $C-5$ & AB307425 & AB544223 \\
\hline BRbv700 & Cattle & Caldas Novas & 2001 & A & $\mathrm{Nd}$ & $\mathrm{Nd}$ & AB544224 \\
\hline BRbv701 & Cattle & Caldas Novas & 2001 & A & $C-5$ & AB307426 & AB544225 \\
\hline BRhr704 & Horse & Corumbaíba & 2001 & A & $\mathrm{Nd}$ & $\mathrm{Nd}$ & AB544227 \\
\hline BRbv705 & Cattle & Ipameri & 2001 & A & $C-5$ & AB307428 & AB544228 \\
\hline BRbv707 & Cattle & Corumbaíba & 2001 & A & $\mathrm{Nd}$ & $\mathrm{Nd}$ & AB544229 \\
\hline BRsp708 & Sheep & Ouvidor & 2001 & A & $\mathrm{Nd}$ & $\mathrm{Nd}$ & AB544230 \\
\hline BRbv710 & Cattle & Morrinhos & 2001 & A & $C-5$ & AB307431 & AB544232 \\
\hline BRbv711 & Cattle & Morrinhos & 2001 & A & $C-5$ & AB307432 & AB544233 \\
\hline BRbv717 & Cattle & Ipameri & 2001 & A & $C-5$ & AB307438 & AB544235 \\
\hline BRbv718 & Cattle & Ipameri & 2001 & A & $C-5$ & AB307439 & AB544236 \\
\hline BRbv741 & Cattle & Marzagão & 2001 & A & $C-5$ & AB307450 & AB544244 \\
\hline BRbv742 & Cattle & Ipameri & 2001 & A & $C-5$ & AB307451 & AB544245 \\
\hline BRbv745 & Cattle & Buriti Alegre & 2001 & A & $C-5$ & AB307454 & AB544248 \\
\hline BRbv747 & Cattle & Corumbaíba & 2001 & A & Ud & AB307456 & AB544250 \\
\hline BRbv748 & Cattle & Campo Alegre de Goiás & 2001 & A & $C-5$ & AB307457 & AB544251 \\
\hline BRbv752 & Cattle & Catalão & 2001 & A & Ud & AB307461 & AB544254 \\
\hline BRhr755 & Horse & Cumari & 2001 & A & $\mathrm{Nd}$ & $\mathrm{Nd}$ & AB544257 \\
\hline BRbv770 & Cattle & Ipameri & 2002 & A & $C-5$ & AB307476 & AB544269 \\
\hline BRbv771 & Cattle & Caldas Novas & 2002 & A & $C-5$ & AB307477 & AB544270 \\
\hline BRbv780 & Cattle & Nova Crixás & 2002 & A & $C-5$ & AB307484 & AB544274 \\
\hline BRbv785 & Cattle & Corumbaíba & 2002 & A & $C-5$ & AB307486 & AB544277 \\
\hline BRbv786 & Cattle & Buriti Alegre & 2002 & A & Ud & AB307487 & AB544278 \\
\hline BRbv796 & Cattle & Pires do Rio & 2002 & A & C-6 & AB307495 & AB544283 \\
\hline BRbv797 & Cattle & Cristalina & 2002 & A & C-6 & AB307496 & AB544284 \\
\hline BRbv402 & Cattle & Divinópolis de Goiás & 2002 & B & $C-5$ & AB307212 & AB544109 \\
\hline BRbv406 & Cattle & Posse & 2002 & B & $C-5$ & AB307216 & AB544113 \\
\hline BRbv459 & Cattle & Monte Alegre de Goiás & 2002 & B & $C-5$ & AB307266 & AB544153 \\
\hline BRbv461 & Cattle & Monte Alegre de Goiás & 2002 & B & $C-5$ & AB307268 & AB544155 \\
\hline BRbv462 & Cattle & Monte Alegre de Goiás & 2002 & B & C-5 & AB307269 & AB544156 \\
\hline BRbv479 & Cattle & Colinas do Sul & 2002 & B & $C-5$ & AB307285 & AB544168 \\
\hline BRbv481 & Cattle & Serranópolis & 2002 & B & C-5 & AB307287 & AB544169 \\
\hline
\end{tabular}


Table 1 Isolates from Goiás (Continued)

\begin{tabular}{|c|c|c|c|c|c|c|c|}
\hline BRbv490 & Cattle & Colinas do Sul & 2002 & B & $C-5$ & AB307296 & AB544175 \\
\hline BRbv494 & Cattle & Divinópolis de Goiás & 2002 & B & $C-5$ & AB307299 & AB544178 \\
\hline BRbv500 & Cattle & Colinas do Sul & 2002 & B & $C-5$ & AB307304 & AB544184 \\
\hline BRbv505 & Cattle & Colinas do Sul & 2002 & B & $C-5$ & AB307309 & AB544188 \\
\hline BRhr506 & Horse & Colinas do Sul & 2002 & B & $\mathrm{Nd}$ & $\mathrm{Nd}$ & AB544189 \\
\hline BRhr507 & Horse & Colinas do Sul & 2002 & B & $\mathrm{Nd}$ & $\mathrm{Nd}$ & AB544190 \\
\hline BRbv510 & Cattle & Monte Alegre de Goiás & 2002 & B & $C-5$ & AB307312 & AB544193 \\
\hline BRbv511 & Cattle & Divinópolis de Goiás & 2002 & B & $C-5$ & AB307313 & AB544194 \\
\hline BRbv518 & Cattle & Campos Belos & 2002 & B & $C-5$ & AB307319 & AB544199 \\
\hline BRbv525 & Cattle & Panamá & 2002 & B & $C-5$ & AB307324 & AB544205 \\
\hline BRbv753 & Cattle & Monte Alegre de Goiás & 2001 & B & $C-5$ & AB307462 & AB544255 \\
\hline BRbv762 & Cattle & Divinópolis de Goiás & 2001 & B & $C-5$ & AB307470 & AB544263 \\
\hline BRbv387 & Cattle & Doverlândia & 2002 & C & $C-12$ & AB307198 & AB544096 \\
\hline BRbv401 & Cattle & Mineiros & 2002 & C & $C-12$ & AB307211 & AB544108 \\
\hline BRbv424 & Cattle & Doverlândia & 2002 & C & $C-12$ & AB307234 & AB544125 \\
\hline BRbv692 & Cattle & Mineiros & 2001 & C & $C-12$ & AB307419 & AB544217 \\
\hline BRbv712 & Cattle & Doverlândia & 2001 & C & $C-12$ & AB307433 & AB544234 \\
\hline BRbv751 & Cattle & Mineiros & 2001 & C & $C-12$ & AB307460 & AB544253 \\
\hline BRbv383 & Cattle & Nova América & 2002 & D & $C-1$ & AB307194 & AB544092 \\
\hline BRbv384 & Cattle & Nova América & 2002 & $D$ & $C-1$ & AB307195 & AB544093 \\
\hline BRbv409 & Cattle & Rubiataba & 2002 & $D$ & $C-1$ & AB307219 & AB544115 \\
\hline BRbv411 & Cattle & Morrinhos & 2002 & D & $C-1$ & AB307221 & AB544117 \\
\hline BRbv452 & Cattle & Rubiataba & 2002 & $\mathrm{D}$ & $C-1$ & AB307259 & AB544148 \\
\hline BRbv767 & Cattle & Rubiataba & 2002 & $\mathrm{D}$ & $C-1$ & AB307474 & AB544267 \\
\hline BRbv793 & Cattle & Urutaí & 2002 & $\mathrm{D}$ & $C-1$ & AB307493 & AB544282 \\
\hline BRbv440 & Cattle & Nova Crixás & 2002 & $E$ & Ud & AB307249 & AB544139 \\
\hline BRbv519 & Cattle & Mundo Novo & 2002 & E & Ud & AB307320 & AB544200 \\
\hline BRhr389 & Horse & Nova Crixás & 2002 & $\mathrm{~F}$ & $\mathrm{Nd}$ & $\mathrm{Nd}$ & AB544098 \\
\hline BRbv434 & Cattle & Itapuranga & 2002 & $\mathrm{~F}$ & Ud & AB307243 & AB544133 \\
\hline BRbv439 & Cattle & Mundo Novo & 2002 & $\mathrm{~F}$ & $C-22$ & AB307248 & AB544138 \\
\hline BRbv488 & Cattle & Carmo do Rio Verde & 2002 & $\mathrm{~F}$ & Ud & AB307294 & AB544173 \\
\hline BRbv749 & Cattle & Nova Crixás & 2001 & $\mathrm{~F}$ & $C-22$ & AB307458 & AB544252 \\
\hline BRbv757 & Cattle & Monte Alegre de Goiás & 2001 & $\mathrm{~F}$ & $C-22$ & AB307465 & AB544259 \\
\hline BRbv763 & Cattle & Aruanã & 2002 & $\mathrm{~F}$ & $C-22$ & AB307471 & AB544264 \\
\hline BRbv773 & Cattle & Aruanã & 2002 & $\mathrm{~F}$ & $C-22$ & AB307479 & AB544272 \\
\hline BRbv774 & Cattle & Mozarlândia & 2002 & $\mathrm{~F}$ & $C-22$ & AB307480 & AB544273 \\
\hline BRsp787 & Sheep & Ipameri & 2002 & $\mathrm{~F}$ & $\mathrm{Nd}$ & $\mathrm{Nd}$ & AB544279 \\
\hline BRbv378 & Cattle & Novo Brasil & 2002 & G & $C-21$ & AB307189 & AB544088 \\
\hline BRbv425 & Cattle & Novo Brasil & 2002 & G & $C-21$ & AB307235 & AB544126 \\
\hline BRbv445 & Cattle & Morrinhos & 2002 & G & Ud & AB307254 & AB544142 \\
\hline BRbv448 & Cattle & Buriti de Goiás & 2002 & G & $C-21$ & AB307256 & AB544144 \\
\hline BRhr467 & Horse & Buriti de Goiás & 2002 & G & $\mathrm{Nd}$ & $\mathrm{Nd}$ & AB544158 \\
\hline BRbv474 & Cattle & Moiporá & 2002 & G & $C-21$ & AB307280 & AB544164 \\
\hline BRbv501 & Cattle & Anicuns & 2002 & G & $C-21$ & AB307305 & AB544185 \\
\hline BRbv515 & Cattle & Córrego do Ouro & 2002 & G & $C-21$ & AB307317 & AB544197 \\
\hline BRbv522 & Cattle & Buriti de Goiás & 2002 & G & $\mathrm{Nd}$ & $\mathrm{Nd}$ & AB544202 \\
\hline BRbv523 & Cattle & Córrego do Ouro & 2002 & G & $C-21$ & AB307322 & AB544203 \\
\hline BRbv764 & Cattle & Córrego do Ouro & 2002 & G & $C-21$ & AB307472 & AB544265 \\
\hline BRbv385 & Cattle & Ivolândia & 2002 & $\mathrm{H}$ & $C-21$ & AB307196 & AB544094 \\
\hline BRbv414 & Cattle & Ivolândia & 2002 & $\mathrm{H}$ & $C-21$ & AB307224 & AB544120 \\
\hline BRbv415 & Cattle & Ivolândia & 2002 & $\mathrm{H}$ & $C-21$ & AB307225 & AB544121 \\
\hline BRbv426 & Cattle & Amorinópolis & 2002 & $\mathrm{H}$ & $C-21$ & AB307236 & AB544127 \\
\hline
\end{tabular}


Table 1 Isolates from Goiás (Continued)

\begin{tabular}{|c|c|c|c|c|c|c|c|}
\hline BRbv427 & Cattle & Ivolândia & 2002 & $\mathrm{H}$ & $C-21$ & AB307237 & AB544128 \\
\hline BRbv431 & Cattle & Palestina de Goiás & 2002 & $\mathrm{H}$ & Ud & AB307240 & AB544131 \\
\hline BRbv437 & Cattle & Goiandira & 2002 & $H$ & Ud & AB307246 & AB544136 \\
\hline BRhr703 & Horse & Ivolândia & 2001 & $H$ & $\mathrm{Nd}$ & $\mathrm{Nd}$ & AB544226 \\
\hline BRbv736 & Cattle & Amorinópolis & 2001 & $\mathrm{H}$ & $C-21$ & AB307446 & AB544242 \\
\hline BRbv744 & Cattle & Amorinópolis & 2001 & $\mathrm{H}$ & $C-21$ & AB307453 & AB544247 \\
\hline BRbv370 & Cattle & Caiapônia & 2002 & 1 & $C-21$ & AB307181 & AB544081 \\
\hline BRbv376 & Cattle & Caiapônia & 2002 & 1 & $C-21$ & AB307187 & AB544086 \\
\hline BRbv377 & Cattle & Bom Jardim de Goiás & 2002 & 1 & $C-21$ & AB307188 & AB544087 \\
\hline BRbv388 & Cattle & Piranhas & 2002 & I & Ud & AB307199 & AB544097 \\
\hline BRbv399 & Cattle & Rio Verde & 2002 & 1 & $C-21$ & AB307209 & AB544106 \\
\hline BRbv403 & Cattle & Piranhas & 2002 & 1 & $C-20$ & AB307213 & AB544110 \\
\hline BRbv420 & Cattle & Piranhas & 2002 & 1 & $C-21$ & AB307230 & AB544123 \\
\hline BRbv430 & Cattle & Buriti Alegre & 2002 & 1 & Ud & AB307239 & AB544130 \\
\hline BRbv435 & Cattle & Bom Jardim de Goiás & 2002 & 1 & $C-20$ & AB307244 & AB544134 \\
\hline BRbv436 & Cattle & Piranhas & 2002 & 1 & Ud & AB307245 & AB544135 \\
\hline BRbv450 & Cattle & Palestina de Goiás & 2002 & 1 & $C-21$ & AB307257 & AB544146 \\
\hline BRbv460 & Cattle & Caiapônia & 2002 & 1 & $\mathrm{C}-20$ & AB307267 & AB544154 \\
\hline BRbv470 & Cattle & Piranhas & 2002 & 1 & $C-20$ & AB307276 & AB544160 \\
\hline BRbv491 & Cattle & Caiapônia & 2002 & I & $C-20$ & AB307297 & AB544176 \\
\hline BRbv499 & Cattle & Bom Jardim de Goiás & 2002 & 1 & $\mathrm{Nd}$ & $\mathrm{Nd}$ & AB544183 \\
\hline BRbv513 & Cattle & Caiapônia & 2002 & 1 & $C-20$ & AB307315 & AB544195 \\
\hline BRbv681 & Cattle & Piranhas & 2001 & 1 & $\mathrm{Nd}$ & $\mathrm{Nd}$ & AB544208 \\
\hline BRbv682 & Cattle & Piranhas & 2001 & 1 & $\mathrm{Nd}$ & $\mathrm{Nd}$ & AB544209 \\
\hline BRbv709 & Cattle & Piranhas & 2001 & 1 & $C-21$ & AB307430 & AB544231 \\
\hline BRbv720 & Cattle & Arenópolis & 2001 & 1 & $C-21$ & AB307440 & AB544237 \\
\hline BRbv721 & Cattle & Arenópolis & 2001 & 1 & $C-21$ & AB307441 & AB544238 \\
\hline BRbv722 & Cattle & Arenópolis & 2001 & I & $C-21$ & AB307442 & AB544239 \\
\hline BRbv734 & Cattle & Arenópolis & 2001 & 1 & $C-21$ & AB307444 & AB544240 \\
\hline BRbv735 & Cattle & Arenópolis & 2001 & 1 & Ud & AB307445 & AB544241 \\
\hline BRbv738 & Cattle & Piranhas & 2001 & 1 & $C-20$ & AB307448 & AB544243 \\
\hline BRbv743 & Cattle & Marzagão & 2001 & 1 & $C-20$ & AB307452 & AB544246 \\
\hline BRbv746 & Cattle & Caiapônia & 2001 & 1 & $C-20$ & AB307455 & AB544249 \\
\hline BRbv756 & Cattle & Arenópolis & 2001 & 1 & $C-21$ & AB307464 & AB544258 \\
\hline BRbv758 & Cattle & Iporá & 2001 & 1 & $C-21$ & AB307466 & AB544260 \\
\hline BRbv759 & Cattle & Diorama & 2001 & 1 & $C-21$ & AB307467 & AB544261 \\
\hline BRbv760 & Cattle & Palestina de Goiás & 2001 & 1 & $C-21$ & AB307468 & AB544262 \\
\hline BRbv765 & Cattle & Caiapônia & 2002 & 1 & $C-20$ & AB307473 & AB544266 \\
\hline BRbv769 & Cattle & Palestina de Goiás & 2002 & I & $C-21$ & AB307475 & AB544268 \\
\hline BRbv772 & Cattle & Arenópolis & 2002 & 1 & $C-21$ & AB307478 & AB544271 \\
\hline BRhr782 & Horse & Palestina de Goiás & 2002 & 1 & $\mathrm{Nd}$ & $\mathrm{Nd}$ & AB544275 \\
\hline BRbv784 & Cattle & Piranhas & 2002 & 1 & $C-20$ & AB307485 & AB544276 \\
\hline BRbv790 & Cattle & Pires do Rio & 2002 & 1 & $C-21$ & AB307490 & AB544280 \\
\hline BRbv508 & Cattle & Porangatatu & 2002 & $J$ & $C-3$ & AB307310 & AB544191 \\
\hline BRbv754 & Cattle & Alvorada do Norte & 2001 & $J$ & $C-3$ & AB307463 & AB544256 \\
\hline BRbv792 & Cattle & Morrinhos & 2002 & $J$ & $C-3$ & AB307492 & AB544281 \\
\hline
\end{tabular}

${ }^{\mathrm{a} U n d e f i n e d ;}{ }^{\mathrm{b}}$ No data; ${ }^{\mathrm{C}}$ Kobayashi et al. (2008) 


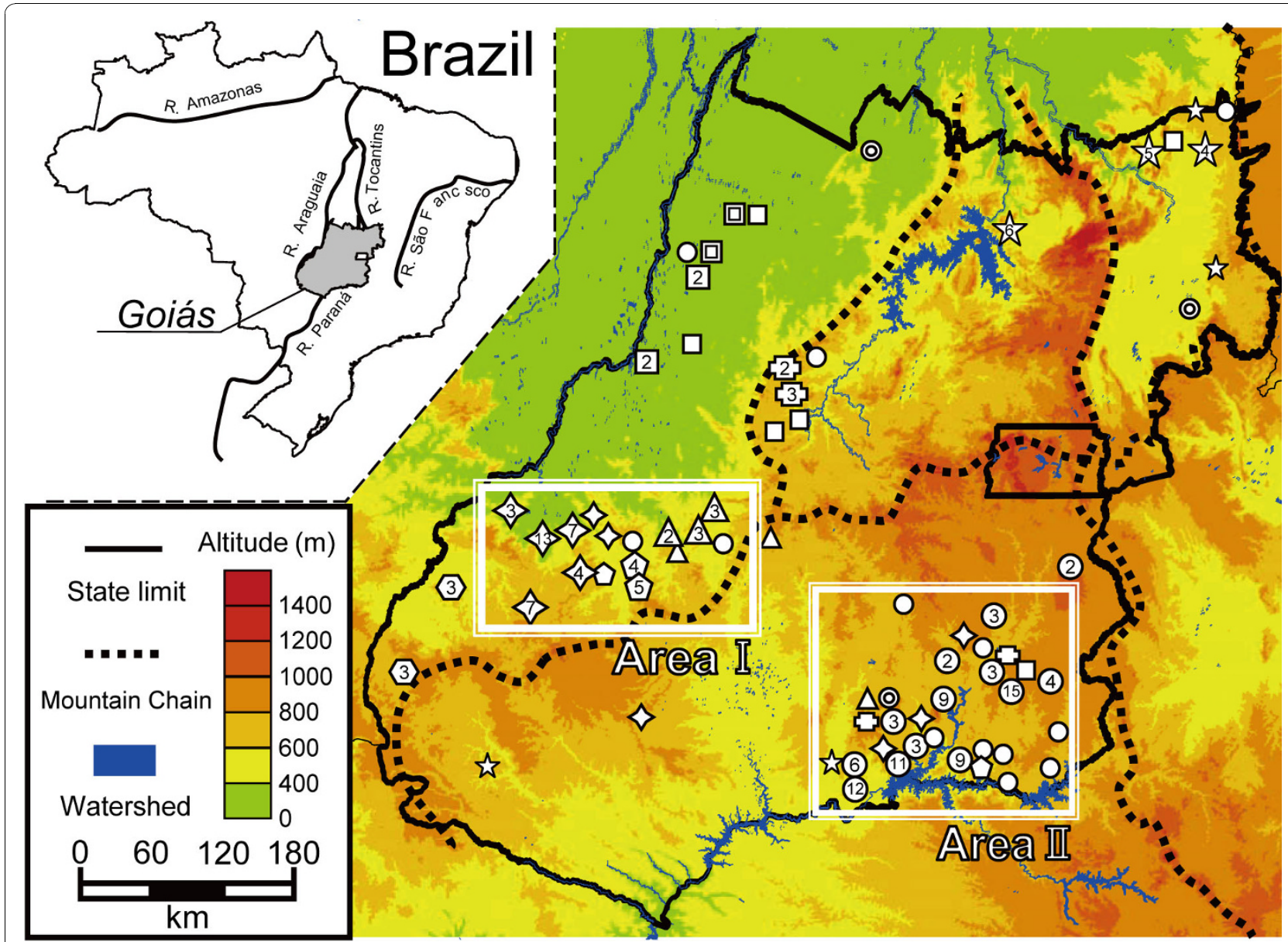

Figure 2 Distribution of vampire bat-transmitted RABV isolates in Goiás. Area I: distribution of G-, H- and I-lineages in mountain ridges. Area II: distribution of A-lineage in the basin. Symbols are same as those used in Fig. 1. Numbers inside the symbols indicate the number of isolates obtained in the same region. Small and open symbols indicated that only one isolate was obtained.

landscape, the results suggest that the distribution patterns of vampire bat-transmitted RABV variants depend on such undulations. On the other hand, the distribution of common vampire bats in a valley is limited by the ridges that form the valley [10], thus supporting the notion that the distribution of RABV variants is affected by smaller topography than mountain chains.

\section{Conclusions}

The present study analyzed the epidemiology of vampire bat-transmitted RABV using a 619-nt region containing the partial glycoprotein gene and the G-L intergenic region, and indicated that the isolates can be further divided into several phylogenetic lineages with significant bootstrap values when compared to characterization based on the 203-nt partial N gene. Furthermore, the phylogenetic lineages were divided by both mountain chains and mountain ridges. In future studies, it will be important to analyze samples from different time points and to elucidate the dynamics of vampire bat- transmitted rabies in order to establish effective and sustainable control measures for preventing rabies circulation among vampire bats.

\section{Methods}

\section{Samples}

A total of 204 samples obtained from 192 cattle, 10 horses and 2 sheep in Goiás from October 2001 to August 2002 were employed, which had been confirmed as rabies positive through fluorescent antibody test and mouse inoculation test (Table 1). Viral RNA was extracted from the brain as described previously [11]. Lineages of the 164 cattle isolates, C-1, C-3, C-5, C-6, $\mathrm{C}-12, \mathrm{C}-20, \mathrm{C}-21$ and $\mathrm{C}-22$, were previously characterized based on a 203-nt sequence of the nucleoprotein gene [5], and are shown in Table 1.

\section{Determination of nucleotide sequences}

RT-PCR and direct sequencing with the HmG5-1302 ${ }_{4619}{ }$ TGTTGAAGTTCACCTYCCMGATGT 4642 , positions 
relative to PV strain genome (Accession No. M13215) and RVLa-1 (5435ATRGGGTCATCATAAACCTC ${ }_{5416}$ ) primer pair were performed as described previously [11]. The target sequence includes the partial glycoprotein gene and G$\mathrm{L}$ intergenic region. Nucleotide sequences were determined using the ATGC program version 4.02 (GENETYX Co., Tokyo, Japan).

\section{Phylogenetioc analysis}

Multiple nucleotide sequence alignments of the partial glycoprotein gene and G-L intergenic region were generated by the ClustalW package in MEGA ver. 4.0 [12]. A phylogenetic tree was constructed by the neighbor joining (NJ) method with bootstrap analysis (1000 psuedoreplicates) under the p-distance model. Phylogenetic clustering supported by a bootstrap value exceeding $70 \%$ was regarded as a reliable lineage [13]. Results were validated by the maximum likelihood method using PhyML [14]. In order to reconfirm the shape of the NJ tree, the ML tree was constructed under HKY substitution model justified by MODELTEST packaged in Hyphy program [15].

\section{Geographical plotting}

The 204 RABV isolates were plotted onto a geographical map described using the DIVA-GIS program [16] with GIS data from Instituto Brasileiro de Geografia e Estatística [17] and DIVA-GIS gData [18].

\section{Acknowledgements}

This work was partly supported by the "Strategic Research Base Development" Program for Private Universities subsidized by the Ministry of Education, Culture, Sports, Science and Technology (MEXT) of Japan.

\section{Author details}

${ }^{1}$ Nihon University Veterinary Research Center, 1866 Kameino, Fujisawa 2520880, Kanagawa, Japan. ${ }^{2}$ Faculty of Agriculture and Veterinary Science, Department of Preventive Veterinary Medicine, UNESP, Via de Acesso Prof. Paulo Donato Castellane, Jaboticabal, São Paulo 14884-900, Brazil. ${ }^{3}$ Department of Preventive Veterinary Medicine and Animal Health, Faculty of Veterinary Medicine and Zootechny, University of São Paulo, Av. Prof. Dr. Orlando Marques de Paiva, 87, Cidade Universtiátria, São Paulo 05508-000, Brazil.

\section{Authors' contributions \\ $\mathrm{SH}$ participated in the design of the study, performed the experimental procedures and the data analysis, and wrote the manuscript. TI, AABC, FHI and TS elaborated the study design, management, coordination, and drafting the manuscript. The authors have read and approved the final manuscript.}

\section{Competing interests}

The authors declare that they have no competing interests.

Received: 26 May 2010 Accepted: 8 November 2010

Published: 8 November 2010

\section{References}

1. Romijn PC, van der Heide R, Cattaneo CA, Silva RC, van der Poel WH: Study of lyssaviruses of bat origin as a source of rabies for other animal species in the State of Rio De Janeiro, Brazil. Am J Trop Med Hyg 2003, 69:81-86.
2. Goncalves MA, Sa Neto RJ, Brazil TK: Outbreak of aggressions and transmission of rabies in human beings by vampire bats in northeastern Brazil. Rev Soc Bras Med Trop 2002, 35:461-464.

3. Mayen F: Haematophagous bats in Brazil, their role in rabies transmission, impact on public health, livestock industry and alternatives to an indiscriminate reduction of bat population. J Vet Med B Infect Dis Vet Public Health 2003, 50:469-472.

4. Massad E, Coutinho FA, Burattini MN, Sallum PC, Lopez LF: A mixed ectoparasite-microparasite model for bat-transmitted rabies. Theor Popul Biol 2001, 60:265-279.

5. Kobayashi Y, Sato G, Mochizuki N, Hirano S, Itou T, Carvalho AA, Albas A, Santos HP, Ito FH, Sakai T: Molecular and geographic analyses of vampire bat-transmitted cattle rabies in central Brazil. BMC Vet Res 2008, 4:44.

6. Arellano-Sota C: Biology, ecology, and control of the vampire bat. Rev Infect Dis 1988, 10:S615-619.

7. Nel LH, Sabeta $C T$, von Teichman B, Jaftha JB, Rupprecht $C E$, Bingham J: Mongoose rabies in southern Africa: a re-evaluation based on molecular epidemiology. Virus Res 2005, 109:165-173.

8. Ngoepe CE, Sabeta C, Nel L: The spread of canine rabies into Free State province of South Africa: A molecular epidemiological characterization. Virus Res 2009, 142:175-180.

9. Kobayashi Y, Ogawa A, Sato G, Sato T, Itou T, Samara SI, Carvalho AA, Nociti DP, Ito FH, Sakai T: Geographical distribution of vampire batrelated cattle rabies in Brazil. J Vet Med Sci 2006, 68:1097-1100.

10. Trajano E: Movements of Cave Bats in Southeastern Brazil, with Emphasis on the Population Ecology of the Common Vampire Bat, Desmodus rotundus (Chiroptera). Biotropica 1996, 28:121-129.

11. Sato G, Itou T, Shoji Y, Miura Y, Mikami T, Ito M, Kurane I, Samara SI, Carvalho AA, Nociti DP, Ito FH, Sakai T: Genetic and phylogenetic analysis of glycoprotein of rabies virus isolated from several species in Brazil. $J$ Vet Med Sci 2004, 66:747-753.

12. Tamura K, Dudley J, Nei M, Kumar S: MEGA4: Molecular Evolutionary Genetics Analysis (MEGA) software version 4.0. Mol Biol Evol 2007, 24:1596-1599.

13. Hills DM, Bull JJ: An empirical test of boostrapping as a method for assessing confidence in phylogenetic analysis. Syst Biol 1993, 42:182-192.

14. Guindon S, Lethiec F, Duroux P, Gascuel O: PHYML Online-a web server for fast maximum likelihood-based phylogenetic inference. Nucleic Acids Res 2005, 33:W557-559.

15. Pond SL, Frost SD, Muse SV: HyPhy: hypothesis testing using phylogenies. Bioinformatics 2005, 21:676-679.

16. Hijmans RJ, Guarino L, Cruz M, Rojas E: Computer tools for spatial analysis of plant genetic resources data: 1. DIVA-GIS. Plant Genet Res News/ 2002, 127:15-19.

17. Instituto Brasileiro de Geografia e Estatística (IBGE). [http://www.ibge.gov. br/servidor_arquivos_geo].

18. DIVA-GIS gData. [http://www.diva-gis.org/gData].

doi:10.1186/1756-0500-3-288

Cite this article as: Hirano et al: Epidemiology of vampire battransmitted rabies virus in Goiás, central Brazil: re-evaluation based on G-L intergenic region. BMC Research Notes 2010 3:288.

\section{Submit your next manuscript to BioMed Central and take full advantage of:}

- Convenient online submission

- Thorough peer review

- No space constraints or color figure charges

- Immediate publication on acceptance

- Inclusion in PubMed, CAS, Scopus and Google Scholar

- Research which is freely available for redistribution 